\title{
Frontières
}

\section{Artifices et beautés sublimes}

\section{Olga Hazan}

Volume 20, numéro 2, printemps 2008

Les musiques et la mort

URI : https://id.erudit.org/iderudit/018361ar

DOI : https://doi.org/10.7202/018361ar

Aller au sommaire du numéro

Éditeur(s)

Université du Québec à Montréal

ISSN

1180-3479 (imprimé)

1916-0976 (numérique)

Découvrir la revue

Citer ce document

Hazan, O. (2008). Artifices et beautés sublimes. Frontières, 20(2), 113-115.

https://doi.org/10.7202/018361ar

Ce document est protégé par la loi sur le droit d'auteur. L'utilisation des services d'Érudit (y compris la reproduction) est assujettie à sa politique d'utilisation que vous pouvez consulter en ligne.

https://apropos.erudit.org/fr/usagers/politique-dutilisation/
Cet article est diffusé et préservé par Érudit.

Érudit est un consortium interuniversitaire sans but lucratif composé de l’Université de Montréal, l'Université Laval et l'Université du Québec à Montréal. Il a pour mission la promotion et la valorisation de la recherche. https://www.erudit.org/fr/ 


\section{Artifices \\ et beautés sublimes}

Olga Hazan

professeure associée, Département d'histoire de l'art, UQAM.

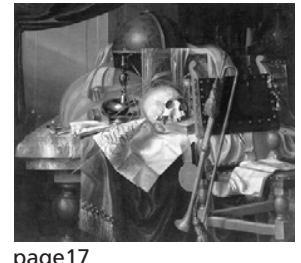

page17

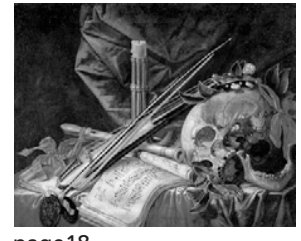

page18

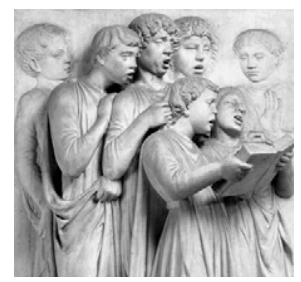

page 24

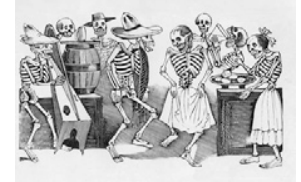

page 79

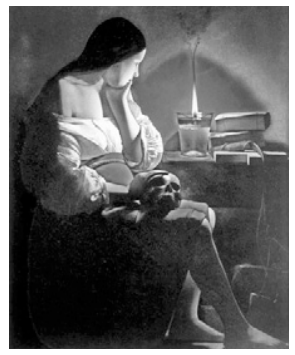

page 9
C'est dans une zone frontière entre la vie et la mort, la mort y étant mise au défi, narguée, conjurée, sublimée par des artifices mimétiques trompeurs et mensongers, que s'inscrit ce dossier d'images, constitué de trois ensembles de quatre, quatre et seize compositions, presque toutes figurées et dont huit ont été créées spécialement pour ce numéro de Frontières par deux artistes et historiens de l'art, Alain Laframboise et Louis Cummins, chacun ayant produit quatre photographies qui seront commentées plus loin. Les seize autres images, s'inscrivant pour la plupart dans la grande tradition européenne, mettent en scène, en des compositions de vastes espaces classiques ou de Gesamkunstwerk baroque, des univers fictifs, aérés ou denses, et minés ça et là de superbes Vanitas en trompe-l'œil (p. 17; p. 18).

Dans cette zone entre vie et mort, hors de l'espace et du temps, se déploient des artifices qui donnent à voir et à ponctuer, par l'évocation d'un geste ou d'un son, des êtres ou des instants qui aujourd'hui ne se voient et ne se mesurent plus. Entre musique et mort, de la musique à la mort et de la musique dans la mort, dans cette zone de fiction orchestrée s'imbriquent ces contrastes, entre vie et mort, tantôt de manière explicite et tantôt, suggestive.

Dans l'ensemble du corpus, l'objet peint ou sculpté sublime la mort, par une évocation de la musique (p. 24) ou de la danse, ou par la suspension du rythme, du geste ou du souffle. Dans quelques cas, la mort se joue au fil du son ou du mouvement, chez la Salomé de Pisano (p. 27), ou dans la gravure mexicaine où dansent les squelettes (p. 79), tandis que d'autres images donnent à voir: la méditation, chez la Madeleine de de La Tour (p. 9); le cri d'effroi devant le corps du Christ mort chez Nicolo dell'Arca (p. 23); le souffle qui s'élève vers Dieu dans le schofar sur la pierre tombale (p. 95); le souffle ultime de la Ludovica Albertoni de Bernini qui agonise (p. 35), ou l'imposition du silence chez Charles Nègre (p. 13). Effroi et pétrification; d'une image à l'autre, on voit graduellement se figer le passage entre vie et mort et s'imposer le silence et l'immobilité, se perdre le souffle.
Ou alors, le temps suspendu se module et se désarticule en moments discontinus, incompatibles, ou en des états, juxtaposés, superposés, contradictoires et mensongers. S'orchestre ainsi le passage au néant, ponctué et décomposé, alors qu'en réalité, le temps se compte déjà à partir du moment de la disparition.

Du point de vue de la narration, s'imposent quelques constantes dans la destinée dramatique de quelques personnages peints ou sculptés - Cécile, Salomé, Jean-Baptiste -, destinée qui chez les deux derniers se joue dans une transposition: de l'archet du violon qui accompagne la demande mortelle de Salomé (p. 27), au fil de l'épée qui occira Jean-Baptiste (p. 27), ou dans les natures mortes, particulièrement propices à évoquer la part d'éphémère dans la vie d'ici-bas. Dans tous ces cas, la facture cruellement belle, concrète, lisse et détaillée des matières, des formes, des textures et des couleurs donne à voir, à entendre, à toucher et à sentir, dans tout son réalisme et tous ses artifices, une mise en scène de la mort par la vie, avec des fruits, des violons, des crânes, des fleurs séchées, des étoffes, du papier, sensations évoquées par des matières dont la précision du détail persistent à rappeler que tout cela, toujours éphémère, aujourd'hui déjà n'existe plus, sinon en pierre sculptée ou en pigment coloré, matières inertes, tous ces protagonistes, héros des légendes et artistes qui leur donnent vie, ayant depuis longtemps rejoint l'univers des défunts.

Destins de femmes - Ludovica, Salomé, Cécile - héroïques et mémorables: Cécile, notamment (p. 91), sainte patronne de la musique, des musiciens, des compositeurs, des luthiers, des chanteurs et des poètes, aveugle aux choses terrestres et qui était si près des cieux qu'elle pouvait entendre les anges chanter; chaste épouse d'un païen du nom de Valérien, condamnée à mort par le gouverneur de Rome, Cécile, qui savait jouer de tous les instruments de musique, mourut face contre terre, les doigts étendus dans la posture que lui donne ici Maderno, et dans laquelle, dit-on, elle fut inhumée avec à ses pied les vêtements ayant essuyé ses plaies. Cécile, dont on célèbre la fête le 22 novembre, présumé jour de 


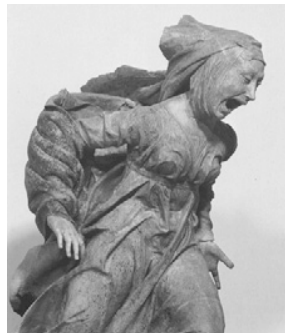

page 23

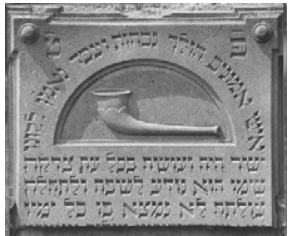

page 95

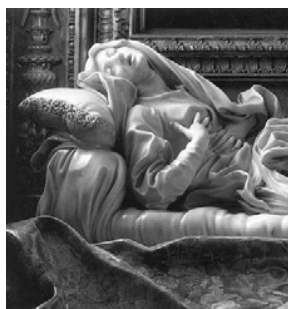

page 35

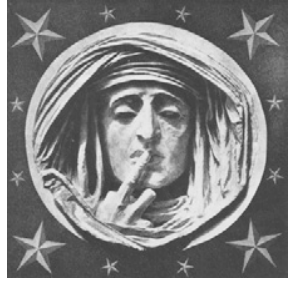

page 13

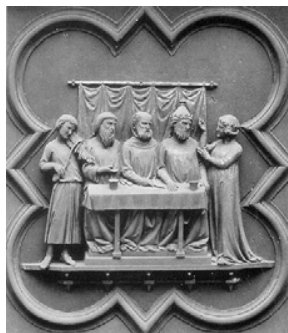

page 27

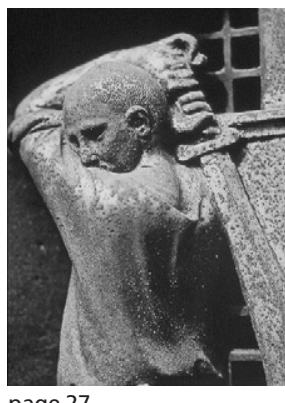

page 27 son décès en l'an 230, aurait inventé l'orgue de petit format dont on retrouve un spécimen en 2007 dans une des quatre photographies d'Alain Laframboise (p. 92).

Se superposent aussi, dans les drames narratifs exposés, des temporalités multiples: de la légende évoquée, puis de l'objet qui encore la donne à voir, par exemple dans la Décapitation de Jean-Baptiste (p. 27), où l'oxydation du bronze trahit le temps qui continue de s'écouler depuis le moment où Andrea Pisano sculptait le geste suspendu du bourreau, dont l'épée ne s'est pas encore abattue, ou encore, dans les pierres tombales, notamment de Chopin et de Berlioz, livrées aux caprices des éléments (p. 95; p. 101 ; p. 102).

Dans d'autres œuvres, le pacte entre musique et mort se fait plus discret, par exemple dans la composition de Vermeer (p. 112), où le temps se module en de multiples modes et registres, comme c'est souvent le cas dans les tableaux de ce peintre, ou dans l'Intérieur avec une femme jouant de l'épinette de De Witte conservé au Musée des beaux-arts de Montréal (p. 3), où de vastes espaces, baignés par la lumière du jour, évoquent la précarité et la menace du Temps, menace de soustraire au regard ce qui, un instant auparavant, s'offrait encore à lui dans toute sa plénitude.

Parmi les diverses stratégies de sublimation à l'œuvre dans l'ensemble de ce corpus on pourrait en identifier quelques-unes (l'espace imparti ne permettant pas d'en discuter plus amplement) : la transposition des genres; la multiplicité et la confusion des temps; les matières déplacées et interverties; l'évocation très précise de diverses sensations; la suspension du temps et des états physiques; la gradation dans l'expression de la vie, du mouvement et la perte du souffle.

Chez Bernini, dans sa Ludovica Albertoni (p. 35), une transposition des matières et une suspension du temps, au centre duquel trône la mort, entre un avant et un après, donne à voir un corps de marbre devant un tableau peint, et la pierre en lieu d'étoffe, dont l'agitation traduit l'émoi du corps et de l'âme. Au mouvement, du corps ou du temps, s'oppose une pétrification, dans la contraction du corps, suspendu entre jouissance et douleur (Ludovica Albertoni, une franciscaine qui s'était ruinée pour prendre soin des pauvres, pratiquait les miracles et était réputée pour ses extases; selon certains, elle pouvait même atteindre à la lévitation; décédée le 31 janvier 1533, elle fut béatifiée par Clément X). Ludovica s'inscrit dans le drame et l'artifice, sa représentation faisant l'objet de transferts et de transpositions des genres, additionnés (sculpture, peinture, architecture).

Multiplicité et confusion des genres, juxtaposition des matières, interverties ou additionnées dans un Gesamkunstwerk qui, en définitive, sert de rhétorique sur le métier d'artiste. Transposition et artifices sont utilisés au bénéfice du créateur d'illusions, lesquelles donnent à voir encore vivant ce qui déjà est mort, et présent ce qui n'est plus visible, comme le voulait Leon Battista Alberti, et immortalise ainsi l'artiste qui défie la mort.
Émane enfin de l'ensemble du corpus: une richesse de la matière, la soie, le marbre, le bois, le papier; une précision des textures et des sensations évoquées, dur, froid, lisse, dense, rugueux, soyeux, froissé, qui se marient et s'opposent, avant et après, dans la pierre froide, la chandelle vacillante, la clarté du jour qui pénètre à travers d'immenses fenêtres. Entre dehors et dedans, entre clarté et pénombre, entre mouvement et fixité, entre chaud et froid, entre hier et demain, entre toi et moi, dans ton visage et dans ton corps, chaud, froid, dans ce qui déjà est souvenir et ce qui, demain, ne sera plus.

$$
* * *
$$

Le corpus photographique d'Alain Laframboise porte un titre à caractère nominatif, voire affirmatif, «IMPRESE», mais qu'il fait suivre d'un point d'interrogation («IMPRESE?»), mettant ainsi en péril la stabilité de ce titre. Dans le commentaire, cité ci-après, qui accompagne ses photographies, Laframboise établit une association aussi contrastée que ce titre, puisqu'elle oppose, d'une part, les stratégies visuelles des images qui, au fil des siècles et des genres, opèrent avec une même constance et, d'autre part, la forme constamment renouvelée des objets où s'inscrit ce processus. Laframboise interroge ainsi la quête de sens que l'on associe aux images, qui souvent résistent à nos certitudes, alors que son corpus photographique traverse quant à lui divers régimes de références, en même temps que le temps qui passe, comme un défi ludique posé aux frontières de la mort.

\section{IMPRESE?}

Sommes-nous affranchis des modèles emblématique et allégorique quand vient le temps de regarder, de penser les images? Les plus belles pages de publicité des revues consacrées à la mode, au design, à toutes les formes actuelles du luxe prouvent bien que non. Un objet ancien, un regard, une attitude, une coiffure, jusqu'aux formes mêmes de la nudité la plus étudiée fonctionnent suivant des procédés rhétoriques millénaires. La personnification, l'allusion, la métonymie, la synecdoque d'individu remontent à la légende homérique et se pratiquent encore aujourd'hui avec la même pertinence et efficacité. Simplement, les aires de signification changent. Le pouvoir des images de représenter, de symboliser et leur capacité expressive traversent les époques. Ainsi un crâne humain sera d'abord une partie du squelette et sur la table de l'anthropologue judiciaire, il restera lié à l'histoire d'une mort particulière; mais ailleurs, dans une fresque médiévale du Campo Santo à Pise ou sur un tombeau baroque du Bernin à Rome, il participera à la personnification de La Mort. Aujourd'hui, en pictogramme sur l'emballage d'un produit toxique, on verra un avertissement du danger que constitue son contenu, et sur l'écu d'un groupe de motards un élément non équivoque d'une héraldique contemporaine qui n'a rien pour rassurer. Combiné à d'autres objets, le crâne se donnera à lire autrement: au pied de la 


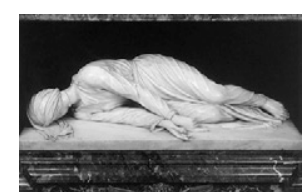

page 91

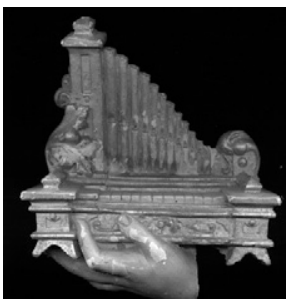

page 92

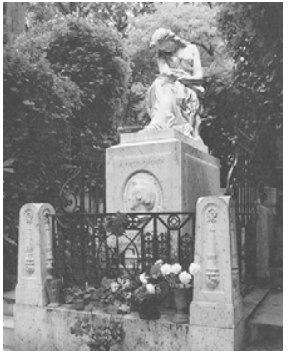

page 101

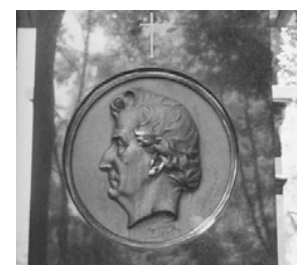

page 102

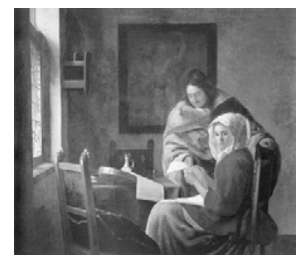

page 112

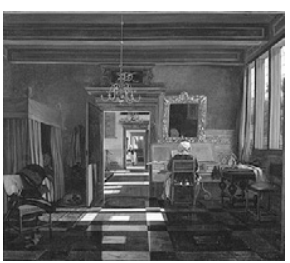

page 3

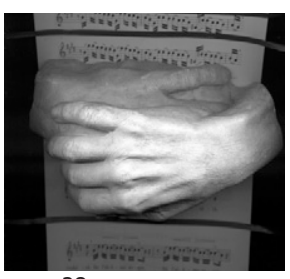

page 28 croix du Christ, dans un tableau du XVII ${ }^{\mathrm{e}}$ siècle, il figurera Adam, mais associé à une guitare électrique, sur l'affiche d'un groupe rock ou dans une photographie de Mapplethorpe ou de Witkin, on y trouvera encore autre chose. Ce que Barthes appelait «un feuilleté de sens », lorsqu'il considérait le plan de la connotation d'une œuvre, se construit et du même coup fuit (s'échappe) par condensation et déplacement ou métonymie et métaphore. Le registre des significations possibles pour chaque figure (image, fragment d'image) n'en finit jamais de s'étendre... Images à caractère d'abord symbolique ou indiciel, qu'importe!

1880-1951 (p. 28) : des dates de naissance et de mort sur le moulage des mains d'un défunt oublié (l'objet n'est plus conservé dans sa famille). Pourtant des empreintes uniques, une histoire unique et rien qui n'ait franchi le cap de l'oubli. Mais la musique et les mots: "fin l'ultimo sospir».

Dans La jeune fille (p. 11), est-ce la Mort ou le crâne qui s'anime et nous regarde? Une mort qui nous apparaît curieusement familière avec ses accessoires contemporains: casque d'écoute, sans doute relié à un iPod, et lunettes solaires (elle sort donc en plein jour?).

Le texte de Notre Enfant chéri (p. 92) figure sur une plaque, vaguement art déco, qui ornait les cercueils d'enfant. La main gauche d'une statue sulpicienne de sainte Cécile porte un objet plus symbolique que réel, un orgue miniature, facilement associable, par son échelle, à un jouet.

Est-ce la mariée duchampienne (trop littéralement) mise à nu par ses célibataires, que l'on retrouve dans Le petit carnaval (page couverture) ou à l'inverse des célibataires trop entreprenants? Poupée, imagerie enfantine, «Carnaval» et "Galop », allusions plus adultes et grivoises. Quel pas faut-il adopter pour cette danse macabre ambiguë?

Les quatre photographies de Louis Cummins donnent à voir deux tandems - le silence et la mort; la musique et la mort - qui se composent et se décomposent en diverses combinaisons, et ce par le biais de transpositions, de transferts et d'enchấnements, de médiums, de matières ou de temps. Les lignes qui suivent ont été rédigées à partir d'une conversation avec l'artiste.

Lacrimosa (p. 109) condense et met en suspens, en accompagnement musical du Requiem de Mozart, un processus de deuil vécu par Cummins après la perte de l'un de ses amis décédé du sida. Prise d'un vidéo, la photographie donne à voir des étincelles: «[...] j'ai brûlé une chandelle qui, au moment de s'éteindre, a fait une flamme très intense, parce que le chandelier de métal dans lequel elle brûlait est devenu très chaud au point d'incendier d'un coup toute la cire qui s'y trouvait». Se trouvent alors condensés dans le geste en suspens de deux ombres qui se quittent, ainsi que dans un transfert d'un médium à l'autre - du geste vivant au film, puis à la photographie - un enchaînement de sensations et de matières en feu, qui se condensent et le / nous consument, jusqu'à la trêve de l'image fixe, où se fige la séparation des deux corps.

Film noir (p. 84), tiré du film de Jules Dassin, Night in the City, rappelle, dans le film, le temps d'arrêt de la musique au moment où des protagonistes sont tués. L'histoire, qui porte sur la boxe, se présente comme une allégorie de la vie moderne, où les petits tentent de devenir grands. Au moment où Harry semble enfin trouver une solution pour rembourser le propriétaire du Club, son créancier et ennemi, l'un et l'autre «jouent de la musique», mais Harry mourra quelques scènes plus loin, parce que l'Establishment ne lui permet pas de se tailler une place parmi les grands.

Les deux autres photographies de Louis Cummins sont plus explicitement politiques. Wagner, «Festspielhaus » (p. 97) rappelle que la résidence de Wagner à Bayreuth demeure aujourd'hui l'un des plus grands centres d'opéra au monde, comme au temps des nazis, où elle servait presque de quartier général de la culture du Reich. La photo est extraite de la vidéo d'une ruine industrielle filmée par Cummins, et qui, à ses yeux, évoque les camps, la destruction et l'absurdité du sublime dans la culture.

$V I H$ / SIDA (p. 87), pour Cummins, « fait référence à la position officielle de l'Église catholique, qui condamne toute forme de protection médicale normale (le condom) contre la transmission du sida, entre autres en Afrique, où 5000 enfants sont infectés quotidiennement». Les directives officielles et les institutions qui les transmettent sont "de véritables agents de destruction génocidaire; un continent meurt, c'est connu de tout le monde, et les autorités religieuses catholiques continuent de défendre des principes contraires à la rationalité scientifique la plus élémentaire».

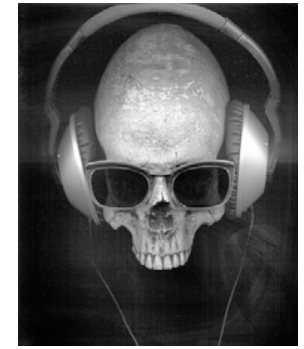

page 11

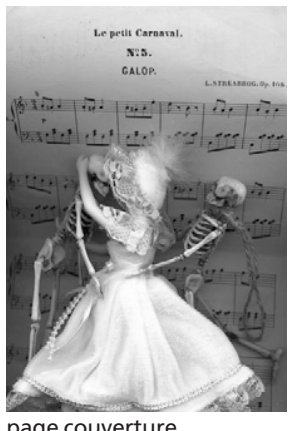

page couverture
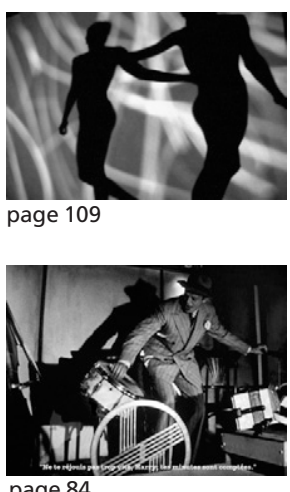

page 84

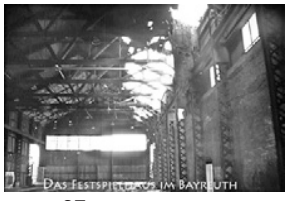

page 97

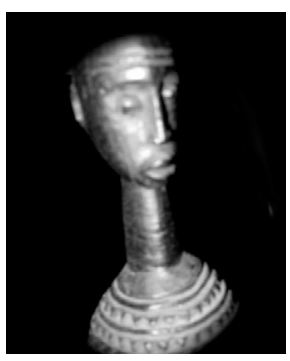

page 87 